\title{
Periodic vortex breakdown in wide spherical gaps
}

\author{
Li Yuan, ${ }^{\text {a) }}$ Dexun Fu, and Yanwen Ma \\ LNM, Institute of Mechanics, Academia Sinica, Beijing 100080, China
}

(Received 24 July 1996; accepted 23 January 1997)

The flow field with vortex breakdown in wide spherical gaps was studied numerically by a finite difference method under the axisymmetric condition. The result shows that the flow bifurcates to periodic motion as the Reynolds number or the eccentricity of the spheres increases. (C) 1997 American Institute of Physics. [S1070-6631(97)03205-4]

Flow between two coaxially rotating spheres has received much attention in the last decades ${ }^{1,2}$ because it is a typical case for studying nonlinear characteristics such as bifurcations and chaos which are often related to the dynamic system of the incompressible Navier-Stokes equations in a closed domain. Depending on different control parameters and time histories of the boundary condition, very different fluid phenomena were observed in narrow to medium gaps $;^{2}$ e.g., Taylor vortex, spiral vortex, wavy toroidal vortex, shear waves, Stuart vortex, quasi-periodic flows and chaotic flows. Recently there have been several studies on the vortical motions in wide spherical gaps. Bar-Yoseph et $a .^{3}$ first reported a numerical study of vortex breakdown in the polar region of a spherical gap in a certain range of the Reynolds number. Their following work ${ }^{4}$ reported further results of vortex breakdown at different Reynolds numbers, gap widths, eccentricities and rotation ratios. Their experimental result indicated that the vortex breakdown might be nonsteady or nonaxisymmetric. In a parallel study of the confined swirling flow in a cylindrical container, Gelfgat et al. ${ }^{5}$ proved that the appearance or disappearance of vortex breakdown in steady states are not connected with the instability of the flow and for a certain aspect ratio range the transition from a steady to an oscillatory flow regime takes place as a result of an axisymmetrical supercritical Hopf bifurcation. This fact that a periodic motion always happens in a flow in a closed geometry with increasing $R e$ number motivates our present numerical study.

We examine numerically the incompressible flow between a rotating inner and a stationary outer sphere. The eccentricity is along the common axis of rotation. There are three control parameters: a dimensionless gap width $s \equiv\left(R_{o}-R_{i}\right) / R_{o}$, a Reynolds number $R e \equiv \Omega_{i} R_{o}^{2} / \nu$ and a dimensionless eccentricity $\epsilon=e /\left(R_{o}-R_{i}\right)$, where $R_{i}$ and $R_{o}$ are the inner and outer radii, $\Omega_{i}$ is the angular velocity of the inner sphere, $e$ the distance between the two sphere centers and $\nu$ the kinematic viscosity. We limit our study to "widegap" geometries, i.e. the gap width $s \approx 0.5$ and the eccentricity is either 0 or 0.9 . For a concentric gap $s=0.55$ at $R e \geqslant 10^{4}$, the numerical result of Ref. 4 showed that the flow evolves a sequence of single flat bubble, two bubbles and the merged bubble structure (see Fig. 4 in Ref. 4). However that was the result of a steady-state solver. It may be an unstable steady solution which is one branch of a supercritical bifurcation. The supercritical unstable steady solution is difficult to remain for a long time even using an unsteady solver, because any infinitestsmal disturbance will destabilize it. Our unsteady simulation reveals that the flow tends to develop into a periodic flow structure at such high $R e$ numbers.

In our numerical code, the unsteady axisymmetric Navier-Stokes equations were solved by the finite difference method. ${ }^{6,7}$ The method is a modified version of the pseudocompressibility method in which a subiteration is performed at each physical time step to drive the pseudocompressibility terms toward zero to satisfy the continuity equation. Because the computation has to run several hundred revolutions of the inner sphere before the flow develops into a stable steady state or periodic state, it is important to keep the numerical viscosity much less than the physical viscosity. A third-order upwind difference ${ }^{7}$ was used. The divergence of velocity was reduced below $10^{-4}$ within 6 subiterations. This quantity is accurate enough for computing most slow evolutionary flows. Although a stretched grid may be useful

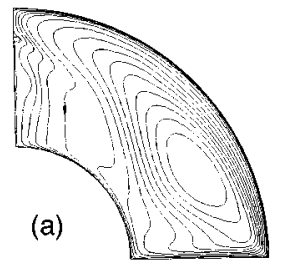

$\mathrm{T}=703.2$

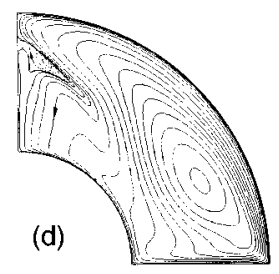

$\mathrm{T}=716.8$

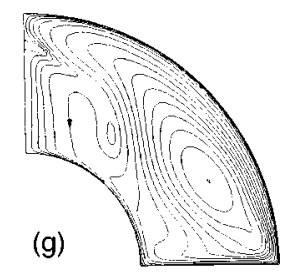

$\mathrm{T}=734$

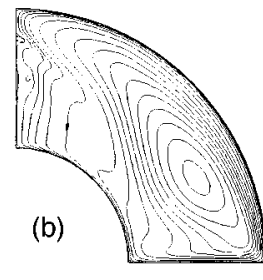

$\mathrm{T}=706.8$

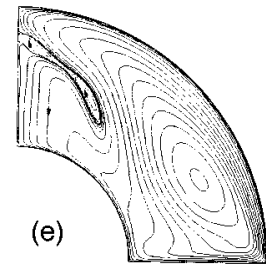

$\mathrm{T}=721.2$

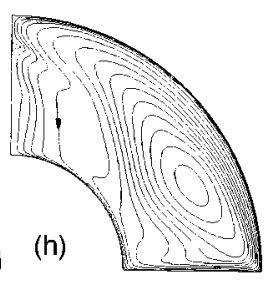

$\mathrm{T}=744$

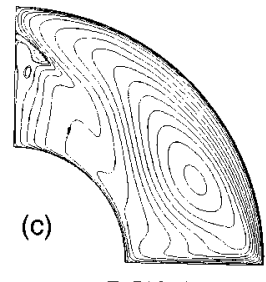

$\mathrm{T}=710,4$

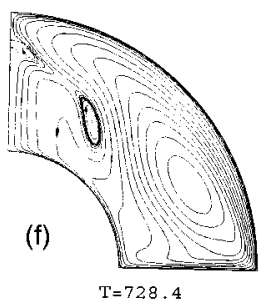

FIG. 1. Temporal variation of the meridional streamline pattern in one period, $s=0.55, R e=12600$, concentric gap. 


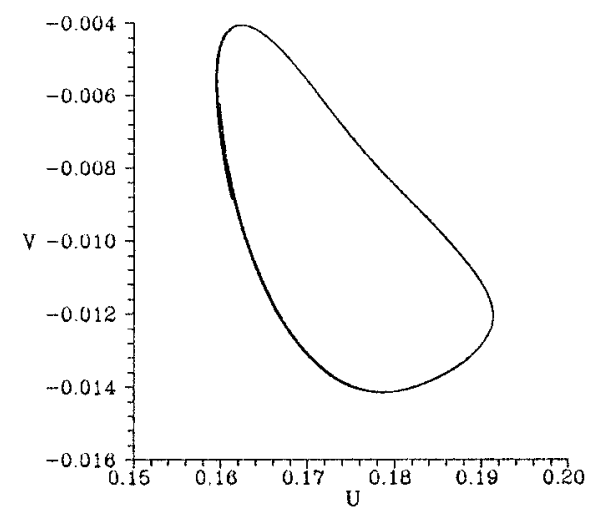

FIG. 2. Phase plot of $(u, v)$ velocity components near the equator at $\left(r-R_{i}\right) /\left(R_{o}-R_{i}\right)=0.4, \theta=80^{\circ}, s=0.55, R e=12600$.

in resolving the boundary layer, the vortex bubble lies in the interior domain and to resolve this region we have to use uniform mesh. The numerical tests showed that the mesh number and time step have significant effects on the computational result. The computations were repeated with three uniform meshes: $81(r) \times 81(\theta), 121 \times 81$ and $141 \times 121$ for a quarter annulus. When mesh number $81 \times 81$ was used, the computed flow for concentric gap $s=0.55, R e=12600$ would have a stationary single vortex bubble but when mesh number $121 \times 81$ and above were used, the result was a periodic flow. In order to obtain a reliable result, mesh number $141 \times 121$ was used. Apart from the mesh number, it is also important to keep the time step small enough so as to make the CFL number less than 1 . In our initial computation with $\Delta t=0.025$, the computed periodic flow had a very large vortex bubble. In the present computation, time step was set to 0.003. Representative results are (a) a concentric gap with $s=0.55$ and $R e=12600$; and (b) an eccentric gap, $s=0.5$, $\epsilon=0.9$ at two $R e$ numbers, $R e=2553$ and $R e=4000$.

Figure 1 shows the time sequence of the meridional streamlines for concentric gap $s=0.55$ at $R e=12600$. We see that a vortex bubble first appears upstream on the polar axis, then grows in size, becomes flat, extends into the inte-

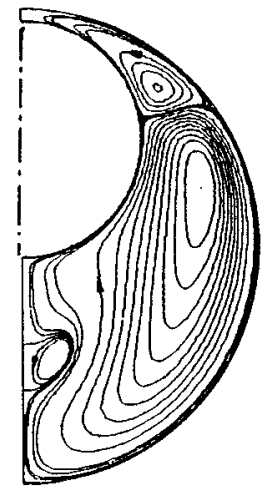

FIG. 3. The meridional streamlines for an eccentric sphere, $\epsilon=0.9, s=0.5$, $R e=2553$.

rior region, and finally splits. The split vortex ring is convected to the interior region and disappears. From Fig. 1(a) to Fig. 1(h) and back to Fig. 1(a), the flow passes through one period. This periodic pattern was obtained after about 150000 time steps with $\Delta T=0.003$, where $T=t \Omega_{i}$. The phase plot (Fig. 2) for $u-v$ velocity components near the equator of the annulus is shown to be a closed loop. This indicates that the flow has become periodic. The result is quite different from the steady two bubble structure in Ref. 4. We think the difference is caused by using different numerical codes. Our unsteady method allows a small numerical disturbance to grow to such an extent as to produce instability of the flow at supercritical Reynolds numbers.

The second case is an eccentric gap $s=0.5, \epsilon=0.9$. The grid number $81 \times 129$ was used. The flow at $R e=2553$ (as given in Table I of Ref. 4) was computed. Our result (Fig. 3) shows that there is only one steady bubble on the wider polar axis rather than a vertically oscillating structure as demonstrated in the experiment. ${ }^{4}$ This difference may be within the expected mismatches of numerics and experiments, or may be due to the fact that a true three-dimensional flow can not be simulated by imposing axisymmetric restriction. However, as $R e$ increases, for example, $R e \geqslant 3000$ in our compu-

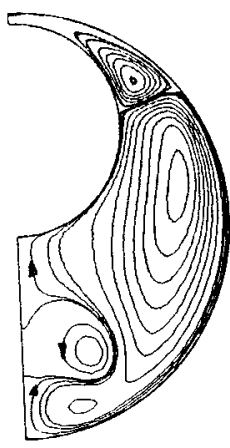

$\mathrm{T}=250$

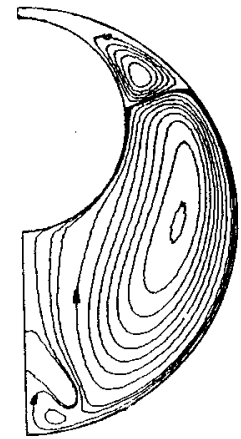

$\mathrm{T}=265$

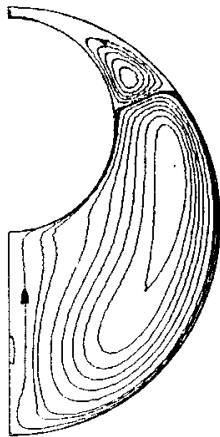

$\mathrm{T}=280$
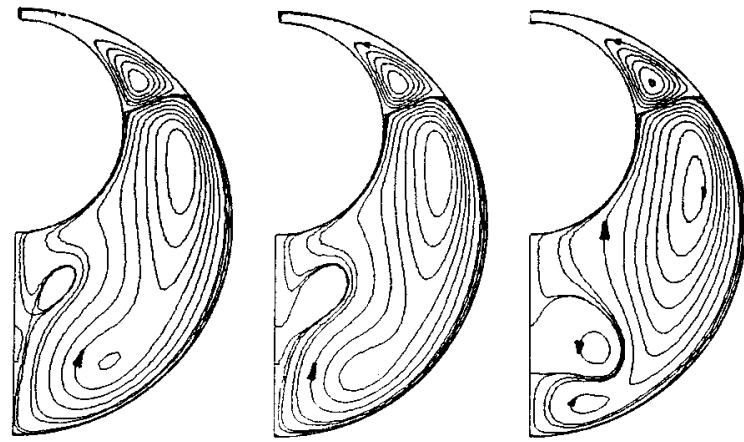

$\mathrm{T}=292$

$$
\mathrm{T}=295
$$

$\mathrm{T}=310$

FIG. 4. Temporal variation of the meridional streamline pattern in one period, $\epsilon=0.9, s=0.5, R e=4000$. 
tation, the flow indeed develops into an axisymmetric periodic one. In Fig. 4, meridional streamlines at different instants show that the vortex bubble swings upstream and downstream in the wider polar region. The period of the velocity on the polar axis is about 9.5 times that of the rotation of the inner sphere. Although direct comparison between the present result and other experimental or numerical ones has not been made, our result seems reasonable and in qualitative agreement with the experimental results of Ref. 4 . The increase of the eccentricity is similar to the increase of the "height/radius ratio", in a closed cylindrical container with a rotating lid, which will promote the appearance of Hopf bifurcation of the flow. ${ }^{4}$ For a real flow, three-dimensional instabilities may occur ahead of the critical Reynolds number or eccentricity where an axisymmetric Hopf bifurcation occurs, therefore a three-dimensional computation is probably necessary to reproduce the experimental results.

\section{ACKNOWLEDGMENTS}

This work was supported by the National Natural Science Foundation of China and the Lab of Scientific and
Engineering Computing, Institute of Computational Mathematics.

a) Author to whom correspondence should be addressed. Electronic mail: lyuan@phy.cuhk.edu.hk

${ }^{1}$ M. Wimmer, "Experiments on a viscous fluid flow between concentric rotating spheres,'” J. Fluid Mech. 78, 317 (1976).

${ }^{2} \mathrm{~K}$. Nakabayashi and Y. Tsuchida, "Flow-history effect on higher modes in the spherical Couette system," J. Fluid Mech. 295, 43 (1995).

${ }^{3}$ P. Bar-Yoseph, S. Seelig, A. Solan, and K. G. Roesner, "Vortex breakdown in spherical gap," Phys. Fluids. 30, 1581 (1987).

${ }^{4}$ P. Bar-Yoseph, K. G. Roesner, and A. Solan, "Vortex breakdown in the polar region between rotating spheres," Phys. Fluids A 4, 1677 (1992).

${ }^{5}$ A. Yu. Gelfgat, P. Z. Bar-Yoseph, and A. Solan, "Stability of confined swirling flow with and without vortex breakdown,' J. Fluid Mech. 311, 1 (1996).

${ }^{6}$ S. E. Rogers and D. Kwak, "Upwind differencing scheme for the time accurate incompressible Navier-Stokes equations," AIAA J. 28, 253 (1990).

${ }^{7}$ L. Yuan, D. Fu, and Y. Ma, "Numerical study of bifurcation solutions of spherical Couette flow," Sci. China Ser. A 39, 187 (1996). 